Supporting information

\title{
Experimental investigation on the dynamics of on-demand ferrofluid drop formation under a pulse-width-modulated non-uniform magnetic field
}

Mohamad Ali Bijarchi ${ }^{\mathrm{a}}$, Mohammad Behshad Shafii ${ }^{\mathrm{a}, *}$

\author{
${ }^{a}$ Mechanical Engineering Department, Sharif University of Technology, Tehran, Iran, P.O. Box: \\ $11155-9567$ \\ *corresponding author: behshad@sharif.edu
}

Number of pages: 3

Number of figures: 2 


\section{A. Number of pulses}

The pulses that are necessary to form a drop by PWM magnetic field changes with $\mathrm{B}_{0}, v$, and also $D$. The number of pulses versus the induced frequency for various duty cycles at $\mathrm{B}_{0}=70 \mathrm{mT}$ and $\mathrm{L}=6 \mathrm{~mm}$ is illustrated in Figure S1(a). The properties in this figure are as same as those, in Figure 9. As can be seen, by intensifying the induced frequency and decreasing the duty cycle, the number of pulses increases. Figure S1(b) shows the effect of magnetic induction on the number of pulses for various duty cycles at $v=20 \mathrm{~Hz}$ and $\mathrm{L}=6 \mathrm{~mm}$. By decreasing $\mathrm{B}_{0}$, the magnetic force becomes weaker and the pulses that are necessary to form a drop would be higher. Also, by reducing the duty cycle, $t_{\text {on }}$ reduces and the number of pulses increases.

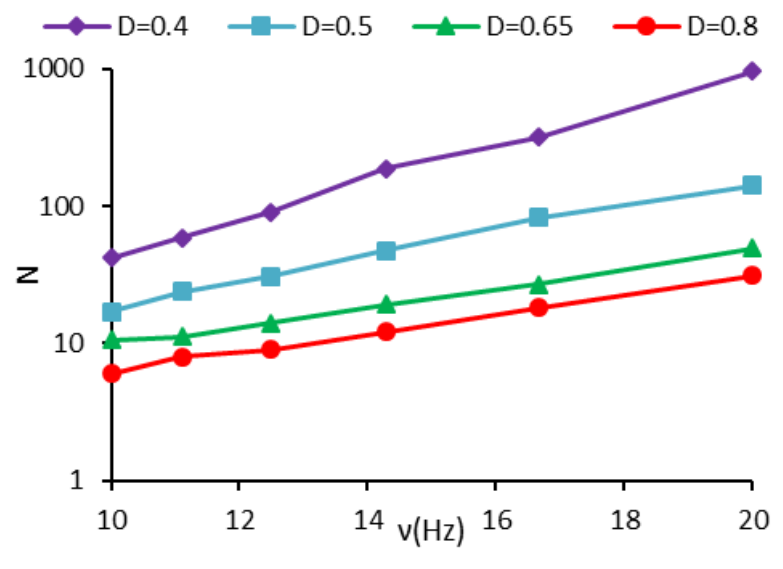

(a)

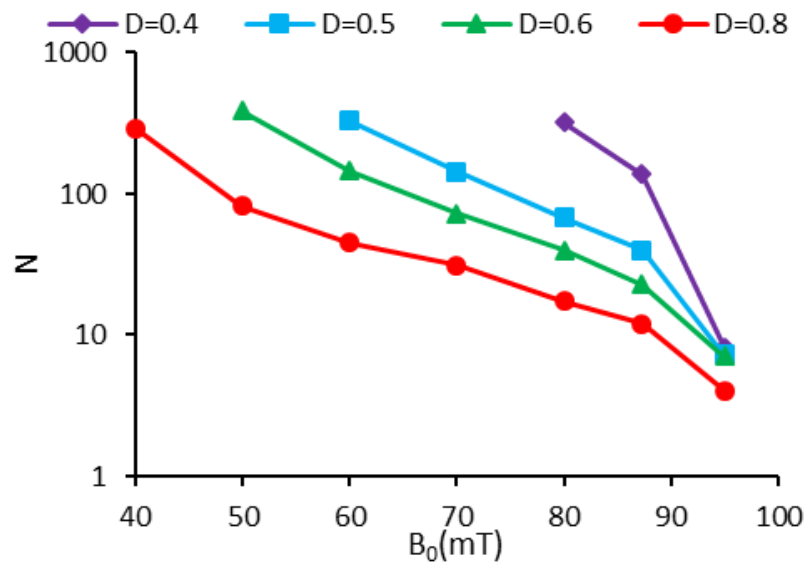

(b)

Figure S1. (a) The pulses that are necessary to form a drop versus induced frequency and constant magnetic induction of $70 \mathrm{mT}$ for various duty cycles and $\mathrm{L}=6 \mathrm{~mm}$, and (b) number of pulses for generation of one drop against magnetic induction and constant induced frequency of $20 \mathrm{~Hz}$, for various duty cycles and $\mathrm{L}=6 \mathrm{~mm}$.

\section{B. The relation of the capillary time scale and the controlling pulse time period}

$T=\sqrt{\rho R^{3} / \sigma}$ is reported as the inertial/capillary time scale in the literature ${ }^{1-2}$. If the ferrofluid droplet with the mass of $m \sim \rho R^{3}$ is considered as a mass box and connected to a spring with the coefficient of surface tension as the stiffness, $T$ represents the magnitude of the oscillation period of a drop when it is stretched, and then released, and it depends on the radius of the drop. As a matter of fact, in PWM-MF, by increasing the number of pulses, more volume of ferrofluid is pulled out of the nozzle. Thus, the mass of the pendant ferrofluid increases and it takes longer time for the ferrofluid to bounce back towards the nozzle. Hence, the capillary time scale increases with pulse repetition until the drop is formed. To investigate the effect of oscillation more deeply, the variation of capillary time scale versus the number of pulses has been shown in Figure S2 based on the total volume of the pendent ferrofluid all around the nozzle measured during the experiments. 


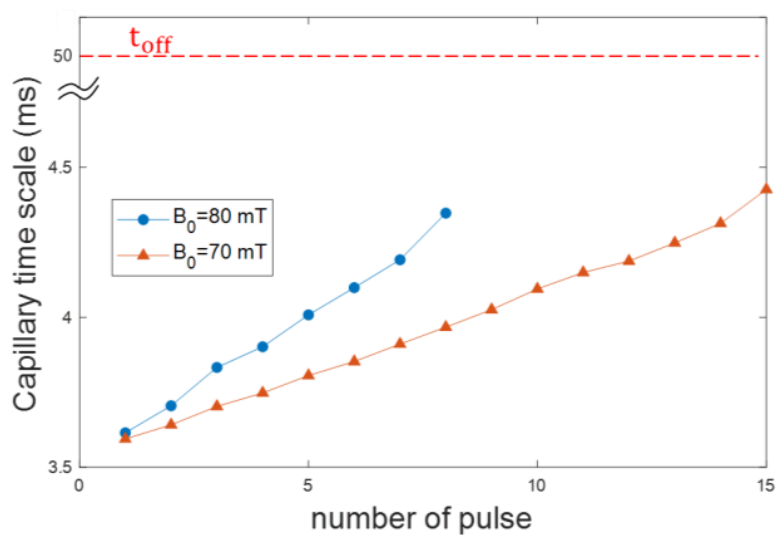

(a)

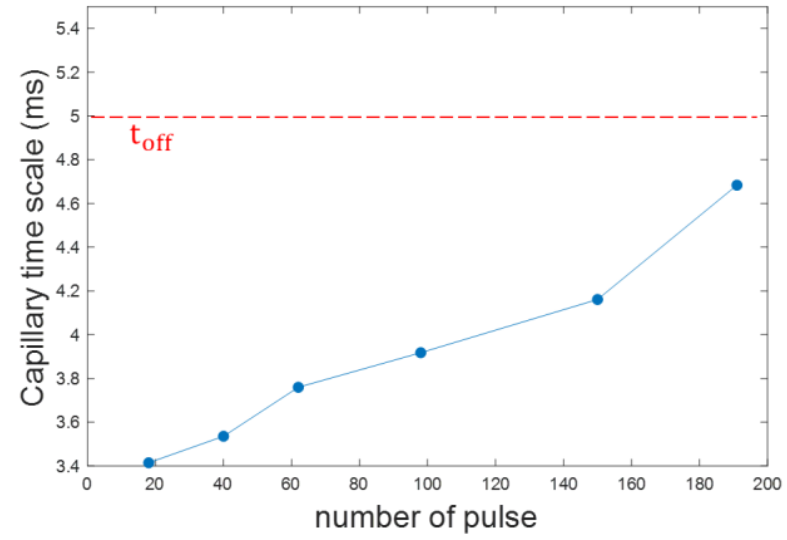

(b)

Figure S2. (a) The variation of capillary time scale versus the number of pulses in the bounce back regime for different magnetic inductions and constant $v=10 \mathrm{~Hz}$, and $\mathrm{D}=0.5$. The maximum value of the capillary time scale is lower than the off-time period $(50 \mathrm{~ms})$. (b) The variation of capillary time scale versus the number of pulses in the hovering regime, considering $v=100 \mathrm{~Hz}$, and $\mathrm{D}=0.5$. The maximum value of the capillary time scale is in the same order of the off-time period $(5 \mathrm{~ms})$.

As can be seen in Figure S2, the capillary time scale increases with the number of pulses in the shown two cases since the volume of the ferrofluid increases. Therefore, once the magnetic field is switched off, the capillary time scale represents the order of time period in which the pendant ferrofluid returns back into and around the nozzle. To have a better sense of different regimes, the off-time period $\left(t_{o f f}=\frac{1}{v}(1-D)\right)$ should be compared with the maximum value of the capillary time scale. In Figure S2(a), the off-time period $(50 \mathrm{~ms})$ is higher than the maximum value of the capillary time scale $(4.5 \mathrm{~ms})$. So, the pendant ferrofluid has enough time to bounce back into and around the nozzle. This phenomenon happens in the bounce back regime. If the induced frequency (v) increases, the oscillations become faster, hence, $\mathrm{t}_{\mathrm{off}}=\frac{1}{v}(1-D)$ decreases to become the same order of magnitude as the capillary time scale. In Figure S2(b) the off-time period (5 ms) has the same order of magnitude as the maximum value of the capillary time scale $(4.7 \mathrm{~ms})$. In this case, with the fast reconnection of the field, the fluid will be pulled again before it has the opportunity to reach the tip of the nozzle. Thus, the droplet formation falls within the hovering regime.

It is worth noting that in Figure S2(a), by increasing magnetic induction, the magnetic force increases and so more volume of ferrofluid is pulled out of the nozzle in each pulse. Therefore, it takes more time for the pending fluid to return back to the nozzle, and the capillary time scale increases.

(1) Biance, A.-L.; Clanet, C.; Quéré, D., First steps in the spreading of a liquid droplet. Physical Review E 2004, 69 (1), 016301.

(2) Ding, H.; Spelt, P. D., Inertial effects in droplet spreading: a comparison between diffuseinterface and level-set simulations. Journal of fluid mechanics 2007, 576, 287-296. 